\title{
The performance of the RADIAL score to predict primary graft failure in heart transplant recipients surviving prolonged ventricular assist device support.
}

Y. Kawamoto, Y. Kusuyama, R. Abe, S. Shibata, Y. Fujino

Osaka University Graduate School of Medicine, Department of Anesthesiology and Intensive Care (Japan)

\section{Background}

Primary Graft Dysfunction (PGD) often occurs intraoperatively and is the primary cause of early mortality after heart transplantation(HT). The RADIAL score is a simple grading system for predicting severe PGD(Table 1). Higher scores are predictive of PGD. However, at our center HT recipients predominately rely on ventricular assist device (VAD) support for exceptionally long periods and the RADIAL score has not been validated in such patients groups.

Table 1 Calculation of the RADIAL score ${ }^{1}$

A point for each category is added for a total score
R Right atrial pressure $>10 \mathrm{mmHg}$,
A recipient Age $>60 y$,
D Diabetes mellitus,
I Inotrope dependence,
A donor Age>30y,
$\mathrm{L}$ Length of ischemic time $>240$

\section{Goal of Study}

Our goal was to examined the performance of the the RADIAL score for predicting PGD at our single center.

\section{Methods}

We conducted a retrospective analysis of 63 adult $\mathrm{HT}$ recipients at our hospital from 2010 to 2016. Collected data included age, sex, reliance and length of VAD support, and the occurrence of PGD. The RADIAL score was calculated for each case. Our primary outcome was the correlation of the RADIAL score with the occurrence of PGD. To assess the predictive value of the RADIAL score a logistic-regression model was constructed from the collected results and the area under a receiver-operating characteristic (ROC) curve was calculated. A value over 0.7 was considered as an acceptable predictive performance model.

\section{Results}

We included $63 \mathrm{HT}$ cases for the final analysis.(Table 2) There were 27 cases of PGD.(Table 3) The RADIAL score was calculated for each case. (Table 3) The average RADIAL score for PGD and non-PGD cases was, $2.4 \pm 1.0$ and $1.9 \pm 1.0$, respectively $(p=0.06)$. The area under the ROC curve calculated from our model was 0.61 .

Table 2 Patient background and severity of Primary Graft Dysfunction (PGD)

\begin{tabular}{|c|c|}
\hline \multicolumn{2}{|c|}{ Patient Background } \\
\hline Number of patients [n] & 63 \\
\hline Age, years & $39 \pm 13$ \\
\hline Sex (female / male) & $22 / 41$ \\
\hline $\mathrm{BMI}\left(\mathrm{kg} / \mathrm{m}^{2}\right)$ & $20.6 \pm$ \\
& 3.4 \\
\hline Circulatory assist & \\
\hline LVAD , $\mathrm{n}(\%)$ & $57(90.5)$ \\
\hline assist period, (days) & $869 \pm 398$ \\
\hline LVAD drive-line & $17(27.9)$ \\
infection , n (\%) & \\
\hline
\end{tabular}

Severity of Primary Graft Dysfunction [n, (\%)]

\begin{tabular}{|c|c|}
\hline none & $\begin{array}{c}36 \\
(57.1)\end{array}$ \\
\hline mild & $9(14.3)$ \\
\hline moderate & $\begin{array}{c}13 \\
(20.6)\end{array}$ \\
\hline severe & $5(7.9)$ \\
\hline
\end{tabular}

1) RADIAL: A novel primary graft failure risk score in heart transplantation. JavierSegovia et al.

The Journal of Heart and Lung Transplantation 2011;644-651.

Table 3 RADIAL scores

\begin{tabular}{|c|c|}
\hline \multicolumn{2}{|c|}{ RADIAL related values $(\mathbf{n}=63$ ) } \\
\hline $\begin{array}{c}\text { Right atrial pressure } \\
{[\mathrm{mmHg}]}\end{array}$ & $7.9 \pm 4.1$ \\
\hline Recipient age [years] & $39 \pm 13$ \\
\hline Diabetes mellitus [n] & 0 \\
\hline $\begin{array}{c}\text { Inotrope dependence [n , } \\
\text { (\%)] }\end{array}$ & $35 \quad(55.6)$ \\
\hline $\begin{array}{c}\text { Estimated donor age } \\
\text { [years] }\end{array}$ & $42 \pm 13$ \\
\hline $\begin{array}{c}\text { Length of ischemic time } \\
{[\text { min] }}\end{array}$ & $217 \pm 45$ \\
\hline RADIAL score & $2.1 \pm 1.1$ \\
\hline
\end{tabular}

\begin{tabular}{|c|c|}
\hline \multicolumn{2}{|l|}{ RADIAL scores $(\mathbf{n})$} \\
\hline Low risk & 3 \\
\hline 0 point & 15 \\
\hline 1 point & \\
\hline Moderate risk & 25 \\
\hline 2 points & \\
\hline High risk & 12 \\
\hline 3 points & 8 \\
\hline 4 points & 0 \\
\hline 5 points & 0 \\
\hline 6 points
\end{tabular}

Fig.1 The relationship between severity of PGD and RADIAL score.

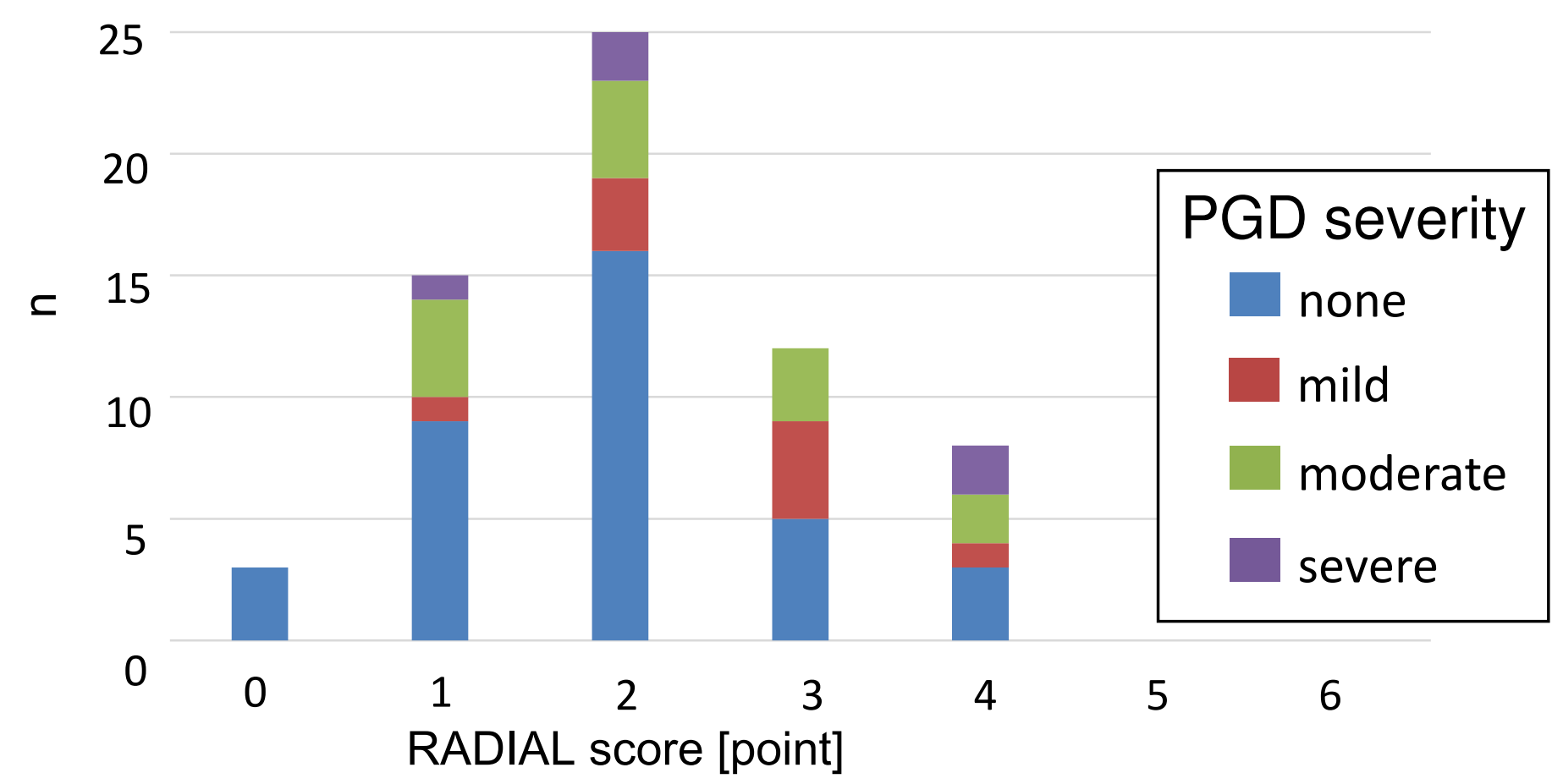

Table 4 Comparison of PGD grades and average RADIAL scores

\begin{tabular}{|c|c|c|r|}
\hline Comparison 1 & PGD of any grade & No PGD & p-value \\
\hline RADIAL score & $2.4 \pm 1.0$ & $1.9 \pm 1.0$ & 0.055 \\
\hline Comparison 2 & $\begin{array}{r}\text { moderate to severe } \\
\text { PGD }\end{array}$ & $\begin{array}{r}\text { Mild PGD and no } \\
\text { PGD }\end{array}$ & p-value \\
\hline RADIAL score & $2.3 \pm 1.1$ & $2.0 \pm 1.0$ & 0.30 \\
\hline Comparison 3 & Severe PGD & $\begin{array}{r}\text { Mild to Moderate PGD } \\
\text { and no PGD }\end{array}$ & p-value \\
\hline RADIAL score & $2.6 \pm 1.3$ & $2.1 \pm 1.0$ & 0.29 \\
\hline
\end{tabular}

Table 5 Performance in predicting PGD severity

\begin{tabular}{|c|c|c|c|c|}
\hline Prediction & $\begin{array}{c}\text { RADIAL } \\
\text { score cut- } \\
\text { off value }\end{array}$ & $\begin{array}{c}\text { Odds } \\
\text { Ratio(95\%C } \\
\text { onfidence } \\
\text { Interval) }\end{array}$ & p-value & $\begin{array}{c}\text { Area Under } \\
\text { Curve from } \\
\text { calculated ROC } \\
\text { curve }\end{array}$ \\
\hline $\begin{array}{c}\text { PGD of any } \\
\text { grade }\end{array}$ & 3 & $0.65(0.36-1.00)$ & 0.06 & 0.63 \\
\hline $\begin{array}{c}\text { moderate to } \\
\text { severe PGD }\end{array}$ & 4 & $0.76(0.45-1.28)$ & 0.29 & 0.56 \\
\hline Severe PGD & 4 & $0.62(0.26-1.50)$ & 0.28 & 0.61 \\
\hline
\end{tabular}

\section{Conclusion}

The performance to for the RADIAL score to predict PGD was low in our model. Although this was a small single center analysis, the RADIAL score may not be useful in predicting $P G D$ in patients undergoing $H T$ after surviving longer periods of VAD support. 